J. Dairy Sci. 95:3125-3130

http://dx.doi.org/10.3168/jds.2011-4769

(C) American Dairy Science Association ${ }^{\circledR}, 2012$.

\title{
Technical note: Quantification of plasma 1- and 3-methylhistidine in dairy cows by high-performance liquid chromatography- tandem mass spectrometry
}

\author{
M. Houweling, ${ }^{* 1}$ S. G. A. van der Drift, $\nmid$ R. Jorritsma, $†$ and A. G. M. Tielens ${ }^{\star}$ \\ *Department of Biochemistry and Cell Biology, Faculty of Veterinary Medicine, Utrecht University, PO Box 80176, 3508 TD Utrecht, \\ the Netherlands \\ †Department of Farm Animal Health, Faculty of Veterinary Medicine, Utrecht University, PO Box 80151, 3508 TD Utrecht, the Netherlands
}

\begin{abstract}
To improve monitoring of protein mobilization in dairy cows, we developed and evaluated a method to quantify 1-methylhistidine and 3-methylhistidine in plasma by HPLC-tandem mass spectrometry. The analytical method described is (1) sensitive: both histidine derivates can be detected in the picomole range; (2) accurate: intra- and interassay coefficients of variation were $<5 \%$ for all standard solutions of 1-methylhistidine and 3-methylhistidine measured (31 to $500 \mathrm{pmol}$ ); (3) specific: 1-methylhistidine is clearly separated from 3-methyl-histidine in plasma samples from dairy cows; and (4) flexible: can be easily adapted to measure other amino acids or compounds containing a primary amine. 1-Methylhistidine is present in plasma of dairy cows at concentrations of $5.0 \pm 1.7 \mu \mathrm{M}$, similar to concentrations of 3-methylhistidine $(4.4 \pm 2.4 \mu M)$. Analytical separation of both histidine metabolites is essential when plasma 3-methylhistidine is used as indicator for muscle breakdown in dairy cows. Specific quantification of the concentration of 3-methylhistidine in bovine plasma samples by HPLC tandem mass spectrometry can improve monitoring of protein mobilization in dairy cows.
\end{abstract}

Key words: 1-methylhistidine, 3-methylhistidine, HPLC-tandem mass spectrometry, protein mobilization

\section{Technical Note}

Modern high-yielding dairy cows enter a state of negative energy balance around parturition when energy demand for maintenance and lactation cannot be met by DMI. To compensate for the high energy demand of lactation, the hormonal balance in cows is set to mobilize fatty acids from adipose tissue (lipolysis) and

Received July 27, 2011.

Accepted January 24, 2012.

${ }^{1}$ Corresponding author: M.Houweling@uu.nl amino acids from muscle protein (proteolysis). Catabolism of the skeletal muscle proteins actin and myosin leads to the release of 3-methylhistidine (3-MH). The plasma concentration of $3-\mathrm{MH}$, a product of the posttranslational methylation of histidine in both actin and myosin, is a good indicator for protein mobilization in cows, because $3-\mathrm{MH}$ is not further metabolized in the body (Harris and Milne, 1981).

Although various techniques to measure 3-MH concentrations in plasma or urine have been described, including use of isotope dilution techniques (Thompson et al., 1996; Lobley et al., 2000) and capillary electrophoresis (Tůma et al., 2005), in most studies samples are derivatized (e.g., with fluorescamine or ophthalaldehyde) and subsequently quantified by HPLC in line with UV or fluorescence detection (Wassner et al., 1980; Qureshi et al., 1984). Using these analytical procedures, 3-MH levels in plasma of dairy cows are elevated around parturition and subsequently decline to basal values around 4 wk after calving (Zurek et al., 1995; Doepel et al., 2002; Akamatsu et al., 2007).

Another methylated derivative of histidine, 1-methylhistidine (1-MH), exists. Anserine ( $\beta$-alanyl-1-methylL-histidine), a dipeptide present in muscle of many animals including cows, is the precursor of 1-MH (Butt and Fleshler, 1965; Dragsted, 2010). 1-Methylhistidine is considered a potential biomarker for meat intake in humans, as anserine is not present in human muscle and its degradation product, 1-MH, is easily detectable in urine (Tůma et al., 2005). Hence, the human body cannot synthesize this methylation product of histidine, and 1-MH can only be produced upon consumption of anserine-containing meat. Interestingly, $1-\mathrm{MH}$ was detected in plasma of steers (Prior and Smith, 1983). Whether anserine breakdown and thus 1-MH production occur in dairy cows has not, to our knowledge, been studied. We hypothesize that breakdown of anserine occurs in cow muscle, resulting in fluctuations in 1-MH release from muscle tissue in cows around parturition. Therefore, the objective was to develop and evaluate a method for plasma analysis of $1-\mathrm{MH}$ and $3-\mathrm{MH}$ by 
HPLC-tandem MS (HPLC-MS/MS) to improve monitoring of protein mobilization in dairy cows.

In addition to standard 1-MH and 3-MH solutions, plasma samples of 24 early lactation dairy cows were analyzed to determine whether cows have significant concentrations of 1-MH after calving and how these relate to $3-\mathrm{MH}$ concentrations during the same period.

The nomenclature commonly used in the literature for methylated histidine molecules will be used throughout this paper, even though this is in conflict with International Union of Pure and Applied Chemistry recommendations. In the literature, the methylated metabolite released upon actin and myosin degradation is always called $3-\mathrm{MH}$ or $\mathrm{N}$-tau-methylhistidine, whereas the product of anserine breakdown is called $1-\mathrm{MH}$.

Stock solutions of $10 \mathrm{mM}$ 1-MH and 3-MH (both compounds were purchased from Sigma-Aldrich, Zwijndrecht, the Netherlands) in $50 \mathrm{~m} M$ phosphate buffer $(\mathrm{pH}=9.0)$ were prepared and stored at $-80^{\circ} \mathrm{C}$ until use. For 1-MH and 3-MH calibration curves, solutions of $50,25,12.5,6.25$, and $3.125 \mu M$ were prepared, of which $50 \mu \mathrm{L}$ was pipetted into conical glass tubes. After derivatization and cleaning (see below), one-fifth of each sample was applied to HPLC-MS/MS for quantification, corresponding to $500,250,125,62.5$, and $31.25 \mathrm{pmol}$, respectively. In total, $1,450 \mu \mathrm{L}$ of $50 \mathrm{mM}$ phosphate buffer $(\mathrm{pH}=9.0)$ was added to $50 \mu \mathrm{L}$ of standard solution and the samples were derivatized by adding $500 \mu \mathrm{L}$ of fluorescamine solution $(3 \mathrm{mg} / 10 \mathrm{~mL}$ of acetone; Sigma-Aldrich). Samples were immediately vortexed, kept at $20^{\circ} \mathrm{C}$ for 5 to $10 \mathrm{~min}$, and subsequently dried in a $40^{\circ} \mathrm{C}$ water bath under a stream of nitrogen. Dried, derivatized samples were then subjected to a cleaning procedure involving homemade columns, which enhanced the sensitivity of the detection by MS/ MS. Columns were prepared by filling the bottom of a 1-mL plastic syringe (without plunger) with a small glass-fiber plug (Boom B.V., Meppel, the Netherlands) and a bed of approximately 0.20 to $0.25 \mathrm{~mL}$ of Davisil 633 18E column material (Grace Alltech, Breda, the Netherlands). Columns were activated by pipetting 0.8 $\mathrm{mL}$ of acetone (liquid chromatography-MS grade, Biosolve, Valkenswaard, the Netherlands) into the syringe followed by centrifugation at $50 \times g$ for $2 \mathrm{~min}$. This centrifugation step was repeated every time a solution was applied to the column. Next, columns were equilibrated by washing twice with approximately $0.8 \mathrm{~mL}$ of $50 \mathrm{~m} M$ phosphate buffer $(\mathrm{pH}=9.0)$. Subsequently, the derivatized samples were dissolved in $0.8 \mathrm{~mL}$ of 50 $\mathrm{m} M$ phosphate buffer $(\mathrm{pH}=9.0)$ and applied to the equilibrated columns.

The columns were washed twice with $0.8 \mathrm{~mL}$ of 50 $\mathrm{m} M$ phosphate buffer and transferred to $15-\mathrm{mL}$ Falcon tubes (the column hangs in the Falcon tube giving a free elution space of $2.5 \mathrm{~mL}$ in the bottom of the tube); samples were collected by eluting the column twice with $0.8 \mathrm{~mL}$ of methanol (liquid chromatography-MS grade, Biosolve). After removing the columns, samples were evaporated in a water bath $\left(40^{\circ} \mathrm{C}\right)$ under a stream of nitrogen. At this point, if necessary, samples could be stored overnight at $5{ }^{\circ} \mathrm{C}$ without loss of signal. The dried, derivatized, cleaned samples were subsequently dissolved in $125 \mu \mathrm{L}$ of $5 \mathrm{mM}$ phosphate buffer $(\mathrm{pH}$ $=9.0$ ) and vortexed for at least $30 \mathrm{~s}$. Samples were transferred to 500- $\mu \mathrm{L}$ Eppendorf tubes and centrifuged for $10 \mathrm{~min}$ at maximum speed $(18,600 \times g)$. After centrifugation, $100 \mu \mathrm{L}$ of each sample was transferred into brown HPLC-MS vials with inserts (Grace Alltech). Vials were capped and placed in the auto sampler of the HPLC-MS/MS device.

Separation of derivatized 1-MH and $3-\mathrm{MH}$ was achieved on a Synergi $4 \mathrm{~mm}$ Max-RP18A column $(250 \times 3 \mathrm{~mm}$; Phenomenex, Torrance, CA $)$. Elution was performed using an HPLC system consisting of 2 HPLC pumps (PE200 series, PerkinElmer, Groningen, the Netherlands) and an auto sampler (PE200 series). The following gradient of water (solution A) and acetonitrile:methanol 1:1 (solution B) was used. From 0 to $2 \mathrm{~min}, 80 \% \mathrm{~A}: 20 \% \mathrm{~B}$; from 2 to $12 \mathrm{~min}$, a linear gradient from $80 \%$ A: $20 \%$ B to $100 \%$ B; from 12 to 16 min, $100 \%$ B; from 16 to $18 \mathrm{~min}$, from $100 \%$ B to $80 \%$ A:20\% B; and finally 6 min at $80 \%$ A:20\% B to equilibrate the column for the next injection. The flow rate was kept constant at $0.4 \mathrm{~mL} / \mathrm{min}$, and $1 \mu M$ serine (for sharper peaks) and $2.5 \mathrm{mM}$ ammonium acetate (to optimize analyte ionization) were used in solutions A and $\mathrm{B}$ as additives. The methylhistidine derivatives were quantified using MS/MS. For quantification, $25-\mu \mathrm{L}$ aliquots were injected into the HPLC-MS/MS, consisting of the above HPLC system coupled to an API-3000 triple quadrupole mass spectrometer, equipped with an electrospray ionization interface. Nitrogen was used as nebulizer and curtain gas. All instruments were purchased from Applied Biosystems (Nieuwerkerk aan den IJssel, the Netherlands). The electrospray ionization interface of the MS was operated at $-4,000 \mathrm{~V}$ and a source temperature of $450^{\circ} \mathrm{C}$. Various traits of the mass spectrometer were optimized using derivatized standards. The entrance, declustering, and focus potential were set at $-10,-50$, and $-250 \mathrm{~V}$, respectively. For quantification, samples were measured in the multiple reaction monitoring mode (also known as "selected reaction monitoring"). The collision energy (CE) was set for each compound separately. The following traces were monitored: $3-\mathrm{MH}$ and $1-\mathrm{MH} ; \mathrm{m} / z \quad 446.2 \rightarrow \mathrm{m} / \mathrm{z}$ 224.0 (at $\mathrm{CE}-35 \mathrm{~V}$ ), $\mathrm{D}_{4}$-ethanolamine; $\mathrm{m} / z 342.1 \rightarrow$ $m / z 176.0$ (at $\mathrm{CE}-23 \mathrm{~V}$ ), and alanine $\mathrm{m} / z 366.1 \rightarrow$ 
$m / z 176.0$ (at CE $-27 \mathrm{~V}$ ). The HPLC-MS/MS instrument was controlled by the Analyst software package (version 1.4.2, Applied Biosystems).
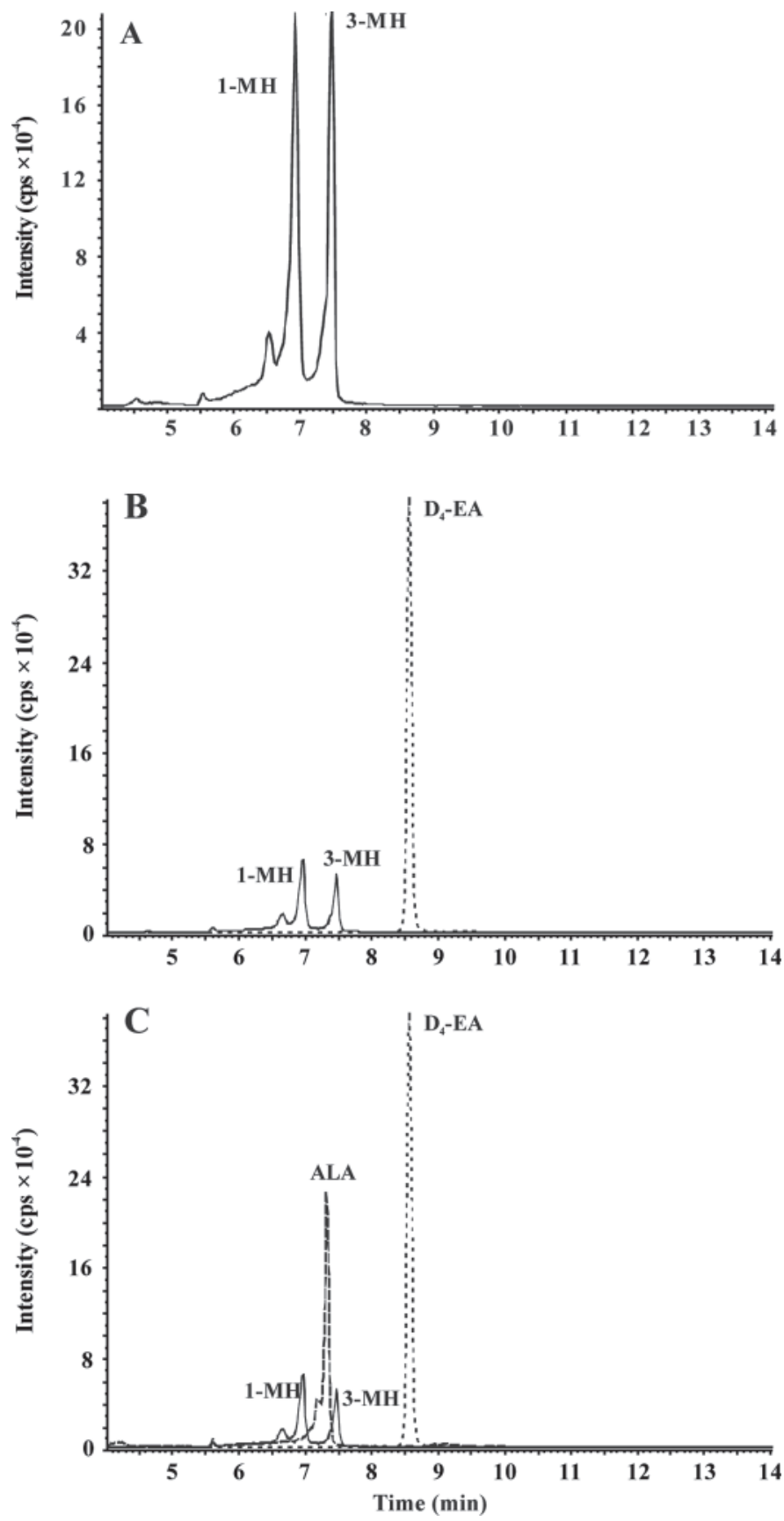

Figure 1. Chromatogram (intensity measured in counts per second, cps) of 1-methylhistidine (1-MH, $m / z 446.2 \rightarrow m / z 224.0)$ and 3-methylhistidine $(3-\mathrm{MH}, m / z 446.2 \rightarrow m / z 224.0)$ standard solution (A) and bovine plasma (B, C), both processed in the presence of the internal standard $\mathrm{D}_{4}$-ethanolamine $\left(\mathrm{D}_{4}\right.$-EA, $m / z 342.1 \rightarrow m / z$ 176.0 $)$. Panel $C$ shows the alanine (ALA, $m / z 366.1 \rightarrow m / z 176.0$ ) signal in bovine plasma.

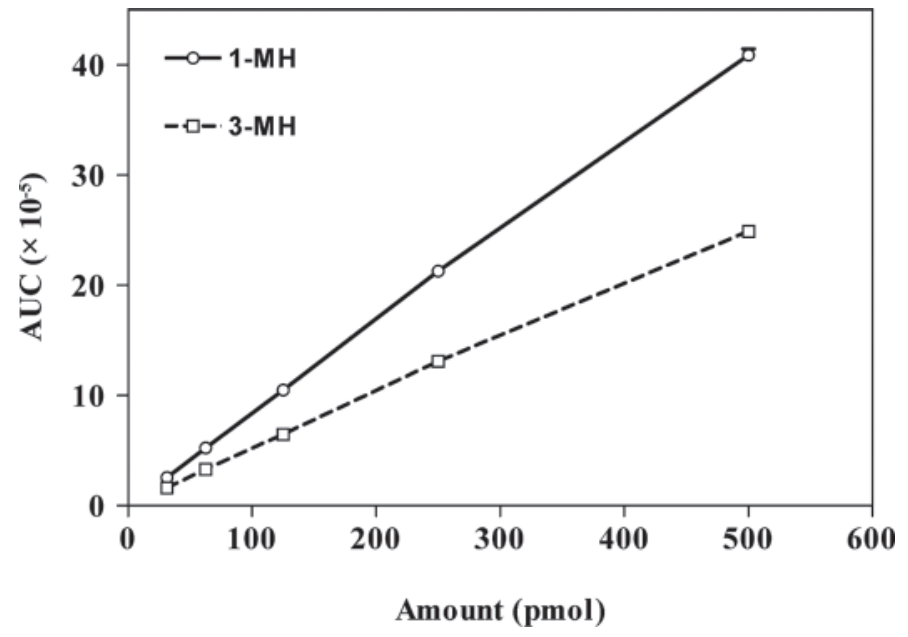

Figure 2. Calibration curves for 1-methylhistidine (1-MH) and 3-methylhistidine (3-MH) based on HPLC-tandem MS analysis of standard solutions. The area under the curve (AUC) is plotted on the y-axis.

Standard solutions at each concentration were processed on 3 different days (triplicate), and each triplicate was subjected 3 times to MS analysis to assess intra- and interassay coefficients of variation $(\mathrm{CV})$ for the analytical procedure, respectively. Heparinized plasma samples from 24 Holstein-Friesian cows of parity $1(\mathrm{n}=6)$, parity $2(\mathrm{n}=6)$, or greater parity $(\mathrm{n}=$ 12) between 5 and 60 DIM were selected for 1-MH and $3-\mathrm{MH}$ analysis. Selected cows originated from different Dutch dairy farms and were sampled in October or November 2010 during a large field study on ketosis (unpublished data), which was evaluated and approved by the Ethical Committee on Animal Experiments from Utrecht University. On the day of sampling, actual milk production (mean $\pm \mathrm{SD}$ ) of cows was $33.4 \pm 8.4 \mathrm{~kg}$, and predicted 305 -d milk yield (mean $\pm \mathrm{SD}$ ) was 8,797 $\pm 1,677 \mathrm{~kg}$. Blood samples were drawn from a coccygeal blood vessel into evacuated heparinized tubes, centrifuged at 2,800 $\times \mathrm{g}$ for $10 \mathrm{~min}$, and plasma was collected and frozen at $-20^{\circ} \mathrm{C}$ until analysis.

Compared with the analytical procedure described for standard solutions, preparation of plasma samples for HPLC-MS/MS analysis involved 2 minor modifications. First, at the start of the procedure, an internal standard $\left(\mathrm{D}_{4}\right.$-ethanolamine; Cambridge Isotope Laboratories, Andover, MA) was added to the samples to correct for the recovery of the analytical procedure. Second, the samples were precleaned by protein precipitation in advance of derivatization with fluorescamine. For protein precipitation, $250 \mu \mathrm{L}$ of heparin plasma and $50 \mu \mathrm{L}$ of $0.1 \mathrm{mM} \mathrm{D}_{4}$-ethanolamine were pipetted into a 2-mL Eppendorf tube, and 1,200 $\mu \mathrm{L}$ of ice-cold 
Table 1. Intra- and interassay coefficients of variation (\%) for HPLC-tandem MS analysis of standard solutions of 1-methylhistidine and 3-methylhistidine and bovine plasma samples

\begin{tabular}{lccccc}
\hline & \multicolumn{2}{c}{ 1-Methylhistidine } & & \multicolumn{2}{c}{ 3-Methylhistidine } \\
\cline { 2 - 3 } \cline { 5 - 6 } Sample & Intraassay & Interassay & & Intraassay & Interassay \\
\hline Standard solution (pmol) & & & & 3.88 & \\
31.25 & 3.11 & 4.58 & & 4.21 & 0.57 \\
62.5 & 3.68 & 2.16 & & 1.60 & 1.67 \\
125 & 2.30 & 3.12 & & 3.31 & 1.17 \\
250 & 3.72 & 2.40 & & 1.86 & 1.68 \\
500 & 3.21 & 2.47 & & 8.08 & 2.85 \\
Bovine plasma & 6.96 & 3.62 & & & \\
\hline
\end{tabular}

$\left(-20^{\circ} \mathrm{C}\right)$ acetone (liquid chromatography-MS grade) was added. Tubes were vortexed, stored at $-20^{\circ} \mathrm{C}$ for at least $60 \mathrm{~min}$, and subsequently centrifuged for 10 min at $18,600 \times g$ at $4^{\circ} \mathrm{C}$ in a refrigerated Eppendorf centrifuge. After centrifugation, the supernatant was transferred into conical glass tubes and dried in a $40^{\circ} \mathrm{C}$ water bath under a stream of nitrogen. At this point, dried, precleaned samples were ready for fluorescamine derivatization, but could also be stored at $-20^{\circ} \mathrm{C}$ for up to 2 wk. From here on, samples were handled as described for the standard solutions. Duplicates of each plasma sample, which were processed on different days, were processed 2 times through the HPLC-MS/ MS device to assess intra- and interassay CV for the analytical procedure. Every time plasma samples were measured, standards of $1-\mathrm{MH}$ and 3-MH were prepared in parallel. Data analysis was performed with Analyst v1.4.1 software (MDS Sciex, Concord, ON, Canada). Intra- and interassay CV were calculated for the analytical procedures of standard solutions and bovine plasma samples.

Differences in plasma 3-MH and 1-MH concentrations and the ratio of $3-\mathrm{MH}$ to $1-\mathrm{MH}$ between periods 0 to 20 DIM, 20 to 40 DIM, and 40 to 60 DIM were analyzed using ANOVA. Additionally, linear regression analysis was used to investigate time trends in plasma $3-\mathrm{MH}$ and 1-MH concentrations between 0 to 20 DIM, the period of most severe protein mobilization in early lactation (Tamminga et al., 1997; Komaragiri et al., 1998; Van Knegsel et al., 2007). The level of significance was set at $P<0.05$.

Figure 1 shows a typical mass spectrum (multiple reaction monitoring mode) of a standard mixture (Figure 1A) and of bovine plasma (Figure 1B). Peaks in bovine plasma samples were identified on basis of their mass, fragmentation, and retention time compared with those of known standards and by spiking the bovine plasma samples before derivatization with known amounts of $1-\mathrm{MH}$ or $3-\mathrm{MH}$ (results of spiking experiments not shown). The recovery for $\mathrm{D}_{4}$-ethanolamine, $1-\mathrm{MH}$, and
3-MH added to samples was similar within the same HPLC-MS/MS run and was approximately $60 \%$.

Figure 1B shows a plasma profile of $1-\mathrm{MH}, 3-\mathrm{MH}$, and the internal standard $\mathrm{D}_{4}$-ethanolamine. Figure $1 \mathrm{C}$ shows that the method described can be easily adapted to quantify other amino acids or other compounds containing a primary amine. As shown in the chromatogram of Figure 1C, alanine eluted next to 1-MH, 3-MH, and $\mathrm{D}_{4}$-ethanolamine. From Figure $1 \mathrm{C}$, it is clear that other compounds present in plasma that contain a primary amine (e.g., amino acids such as alanine) elute from the HPLC-column at a similar retention time as the methylhistidines. A major advantage of this HPLCMS/MS method over HPLC with UV detection is that by using the mass spectrometer as detector, 1-MH and $3-\mathrm{MH}$, once separated by the column, can each be specifically quantified in the presence of other overlapping or co-eluting peaks on the basis of their mass and fragmentation pattern (compare Figure 1B with 1C). Experiments were performed to see whether the presence of alanine influenced the $1-\mathrm{MH}$ and $3-\mathrm{MH}$ signal. When various amounts of alanine (up to 10 times the amount detected in cow plasma) were added to $1-\mathrm{MH}$ and $3-\mathrm{MH}$ standards, no difference in intensity (counts per second, cps) was seen between $1-\mathrm{MH}$ and $3-\mathrm{MH}$ standards without alanine or standards spiked with the highest amount of alanine tested (data not shown).

Calibration curves for 1-MH and 3-MH were produced based on HPLC-MS/MS analysis of standard solutions with increasing concentrations of both methylated histidine metabolites (Figure 2). Although calibration curves of column-cleaned standard solutions are shown, similar results were obtained with noncleaned standards. Intra- and interassay $\mathrm{CV}$ were $<5 \%$ for all standard solutions of 1-MH and 3-MH (Table 1), which indicated that the precision of the analytical procedure was satisfactory. Intraassay CV for HPLC-MS/ MS analysis of bovine plasma samples (Table 1) were slightly greater than those for standard solutions, but still $<10 \%$ and thus considered satisfactory (Murray 
and Lawrence, 1993; Reynal and Broderick, 2009). Interassay CV for plasma analysis of $1-\mathrm{MH}$ and $3-\mathrm{MH}$ were $<5 \%$ (Table 1 ).
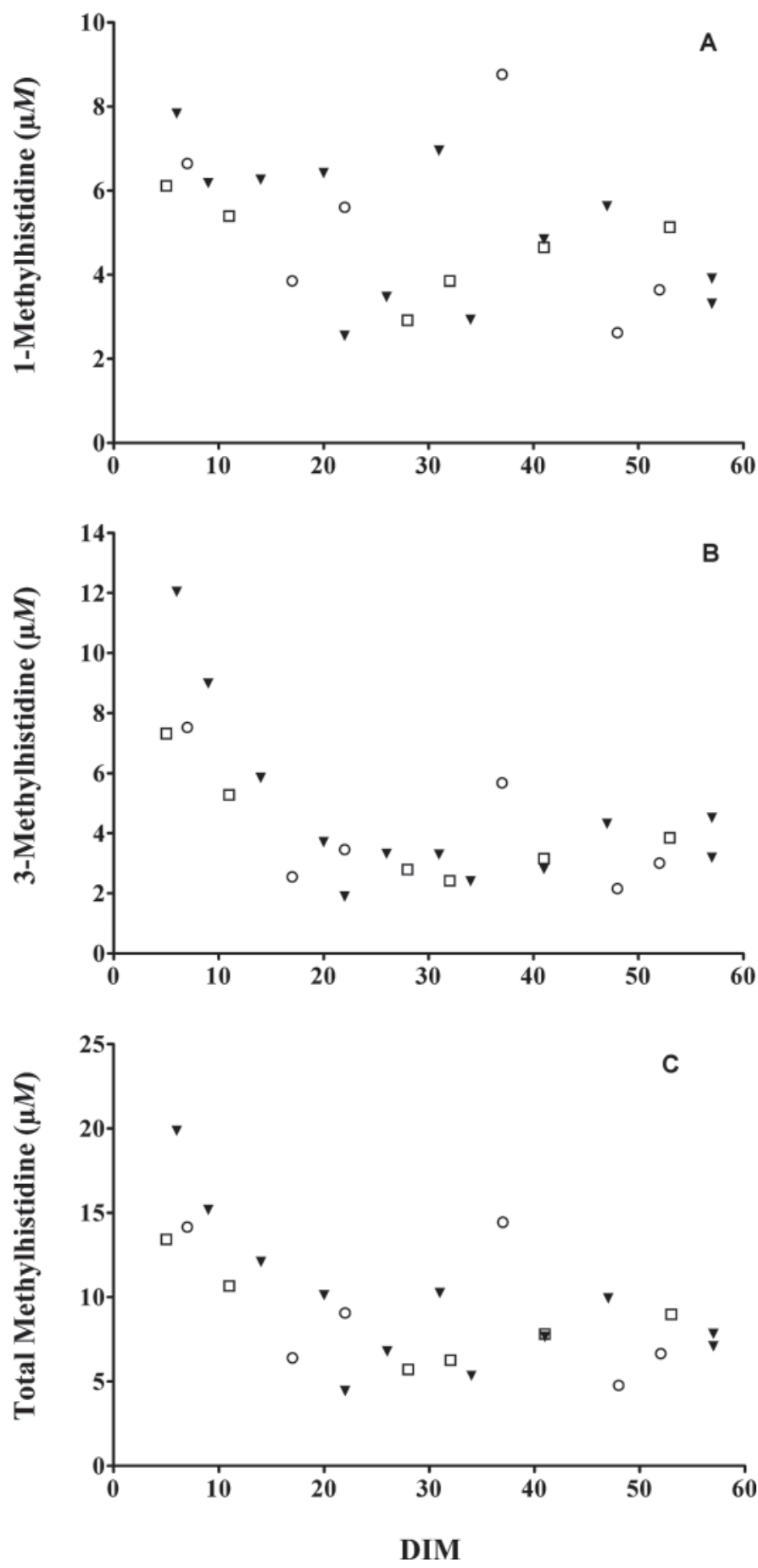

Figure 3. Concentrations of (A) 1-methylhistidine, (B) 3-methylhistidine, and $(\mathrm{C})$ total methylhistidine $(1-\mathrm{MH}+3-\mathrm{MH})$ in plasma of individual cows of parity 1 (squares, $\mathrm{n}=6)$, parity 2 (circles, $\mathrm{n}=6$ ), and greater parities (closed triangles, $\mathrm{n}=12$ ) between 5 and 60 DIM.
The HPLC-MS/MS analysis of plasma samples showed that cows have substantial amounts of $1-\mathrm{MH}$ in early lactation; that is, during protein mobilization (see Figures $1 \mathrm{~B}$ and $3 \mathrm{~A}$ ). Concentrations of 1-MH and $3-\mathrm{MH}$, as well as the total concentrations of both methylhistidine derivatives, in plasma of individual cows are shown in Figure 3.

In early lactation cows, the plasma concentration of $1-\mathrm{MH}$ ranged from 2.55 to $8.76 \mu \mathrm{M}$ and that of $3-\mathrm{MH}$ ranged from 1.90 to $12.04 \mu \mathrm{M}$. Plasma $3-\mathrm{MH}$ concentrations were higher in cows sampled between 0 and 20 DIM compared with cows sampled between 20 and 40 DIM and between 40 and 60 DIM $(P<0.01)$, whereas plasma 1-MH concentrations did not differ between periods. The ratio of plasma $3-\mathrm{MH}$ to $1-\mathrm{MH}$ tended to be higher $(P=0.06)$ in cows between 0 and 20 DIM compared with cows sampled between 20 and 40 DIM. Plasma 3-MH concentrations linearly decreased between 0 and 20 DIM $(P<0.05)$, whereas this time trend was not observed for plasma 1-MH concentrations.

Hydrolysis of the dipeptide anserine by the enzyme carnosinase in serum and tissue results in the formation of 1-MH in humans (Abe et al., 1993; Kubomura et al., 2009). The absence of anserine in human muscle makes it a good biomarker for meat intake by humans. Although substantial amounts of anserine are present in beef muscle (Zapp and Wilson, 1938) and 1-MH is present in steer plasma (Prior and Smith, 1983), it is unknown how hydrolysis of anserine and the subsequent appearance of 1-MH in blood and urine are regulated in cattle. Anserine cannot originate from an external source in cows, as animal by-products are not allowed and not present as ingredients in dairy cattle feeds (European Union 2000/766/EG). Using HPLC-MS/ $\mathrm{MS}$, the amount of 1-MH was detected and quantified in plasma of dairy cows. From the current study, no conclusions can be drawn about the mechanism regulating the appearance of 1-MH in blood of dairy cows. Previous studies that calculated protein balances in cows after calving (Tamminga et al., 1997; Komaragiri et al., 1998; van Knegsel et al., 2007) showed that protein balance in cows was most negative directly after calving and became positive around the fourth week of lactation, which is supported by the observed profile of plasma $3-\mathrm{MH}$ concentrations in this study (Figure 3B), but not by plasma 1-MH concentrations (Figure $3 \mathrm{~A})$. Therefore, our study suggests that plasma $3-\mathrm{MH}$ concentrations specifically, and not $1-\mathrm{MH}$ or total methylhistidine concentrations, should be used as an indicator for muscle proteolysis in dairy cows. Figure 3 shows that the concentrations of $1-\mathrm{MH}$ and $3-\mathrm{MH}$ were similar: $5.0 \pm 1.7$ and $4.4 \pm 2.4 \mu M$, respectively. The latter implies that, when the $3-\mathrm{MH}$ concentrations in 
blood are used as a measure for protein mobilization in dairy cows, it becomes essential to separate these 2 histidine metabolites. The analytical procedure described here was able to differentiate between histidine metabolites and may be preferably applied for 3-MH analysis in future bovine studies.

In conclusion, the developed and validated HPLCMS/MS procedure accurately quantified concentrations of both 1-MH and 3-MH in bovine plasma samples. The achieved separation of the 2 histidine metabolites, and thus the specific quantification of 3-MH, will improve the monitoring of protein mobilization in dairy cows.

\section{ACKNOWLEDGMENTS}

The authors thank Jos Brouwers and Chris van de Lest (Department of Biochemistry and Cell Biology, Faculty of Veterinary Medicine, Utrecht University, Utrecht, the Netherlands) for their technical assistance with the mass spectrometric analysis.

\section{REFERENCES}

Abe, H., E. Okuma, H. Sekine, A. Maeda, and S. Yoshiue. 1993. Human urinary excretion of L-histidine-related compounds after ingestion of several meats and fish muscle. Int. J. Biochem. 25:1245-1249.

Akamatsu, H., Y. Saitoh, M. Serizawa, K. Miyake, Y. Ohba, and K. Nakashima. 2007. Changes of serum 3-methylhistidine concentration and energy-associated metabolites in dairy cows with ketosis. J. Vet. Med. Sci. 69:1091-1093.

Butt, J. H., and B. Fleshler. 1965. Anserine, a source of 1-methylhistidine in urine of man. Proc. Soc. Exp. Biol. Med. 118:722-725.

Doepel, L., H. Lapierre, and J. J. Kennelly. 2002. Peripartum performance and metabolism of dairy cows in response to prepartum energy and protein intake. J. Dairy Sci. 85:2315-2334.

Dragsted, L. O. 2010. Biomarkers of meat intake and the application of nutrigenomics. Meat Sci. 84:301-307.

Harris, C. I., and G. Milne. 1981. The urinary excretion of $N$-taumethyl histidine by cattle: Validation as an index of muscle protein breakdown. Br. J. Nutr. 45:411-422.

Komaragiri, M. V. S., D. P. Casper, and R. A. Erdman. 1998. Factors affecting body tissue mobilization in early lactation dairy cows. 2 .
Effect of dietary fat on mobilization of body fat and protein. J. Dairy Sci. 81:169-175.

Kubomura, D., Y. Matahira, A. Ayano, and H. Matsuda. 2009. Intestinal absorption and blood clearance of L-histidine-related compounds after ingestion of anserine in humans and comparison to anserine-containing diets. J. Agric. Food Chem. 57:1781-1785.

Lobley, G. E., K. D. Sinclair, C. M. Grant, L. Miller, D. Mantle, A. G. Calder, C. C. Warkup, and C. A. Maltin. 2000. The effects of breed and level of nutrition on whole body and muscle protein metabolism in pure-bred Aberdeen Angus and Charolais beef steers. Br. J. Nutr. 84:275-284.

Murray, A., and G. P. Lawrence. 1993. How should the repeatability of clinical measurements be analysed? An assessment of analysis techniques with data from cardiovascular autonomic function tests. Q. J. Med. 86:831-836.

Prior, R. L., and S. B. Smith. 1983. Role of insulin in regulating amino acid metabolism in normal and alloxan-diabetic cattle. J. Nutr. 113:1016-1031.

Qureshi, G. A., S. van den Berg, A. Gutierrez, and J. Bergström. 1984. Determination of histidine and 3-methylhistidine in physiological fluids by high-performance liquid chromatography. J. Chromatogr. 297:83-89.

Reynal, S. M., and G. A. Broderick. 2009. Technical note: A new highperformance liquid chromatography purine assay for quantifying microbial flow. J. Dairy Sci. 92:1177-1181.

Tamminga, S., P. A. Luteijn, and R. G. M. Meijer. 1997. Changes in composition and energy content of liveweight loss in dairy cows with time after parturition. Livest. Prod. Sci. 52:31-38.

Thompson, M. G., R. M. Palmer, A. Thom, K. Garden, G. E. Lobley, and G. Calder. 1996. $\mathrm{N}^{\top}$-methylhistidine turnover in skeletal muscle cells measured by GC-MS. Am. J. Physiol. 270:C1875-C1879

Tůma, P. E. Samcová, and P. Balínová. 2005. Determination of 3-methylhistidine and 1-methylhistidine in untreated urine samples by capillary electrophoresis. J. Chromatogr. B Anal. Technol. Biomed. Life Sci. 821:53-59.

van Knegsel, A. T. M., H. Van Den Brand, J. Dijkstra, W. M. Van Straalen, M. J. W. Heetkamp, S. Tamminga, and B. Kemp. 2007. Dietary energy source in dairy cows in early lactation: Energy partitioning and milk composition. J. Dairy Sci. 90:1467-1476.

Wassner, S. J., J. L. Schlitzer, and J. B. Li. 1980. A rapid, sensitive method for the determination of 3-methylhistidine levels in urine and plasma using high-pressure liquid chromatography. Anal. Biochem. 104:284-289.

Zapp, J. A. Jr., and D. W. Wilson. 1938. Quantitative studies of carnosine and anserine in mammalian muscle. J. Biol. Chem. 126:1927.

Zurek, E., G. R. Foxcroft, and J. J. Kennelly. 1995. Metabolic status and interval to first ovulation in postpartum dairy cows. J. Dairy Sci. 78:1909-1920. 